EPJ Web of Conferences 107, 07004 (2016)

DOI: $10.1051 /$ epjconf/201610707004

(C) Owned by the authors, published by EDP Sciences, 2016

\title{
Alpha-decay and spontaneous fission half-lives of super-heavy nuclei around the doubly magic nucleus ${ }^{270} \mathrm{Hs}$
}

\author{
I. Silisteanu ${ }^{1, a}$ and C. I. Anghel ${ }^{1,2, b}$ \\ ${ }^{1}$ Horia Hulubei National Institute of Physics and Nuclear Engineering, Bucharest-Magurele, RO-077125, Romania \\ ${ }^{2}$ University of Bucharest, Faculty of Physics, Bucharest-Magurele, RO-077125, Romania
}

\begin{abstract}
The $\alpha$-decay and spontaneous fission half-lives of nuclei around ${ }^{270} \mathrm{Hs}$ are calculated with formulas derived from an in-depth analysis of the available experimental data and of results of theoretical models. The parameters of these formulas result from the fit of half-lives with respect to the reaction energies, the height of the SF barrier and the fissionability. The calculated partial and total half-lives are compared with the experimental data and results of other approximations. Half-life predictions are made for many unknown nuclei.
\end{abstract}

\section{Introduction}

The quantity and quality of experimental data on the production and decay of the SHN have increased considerably in the last two decades [1-13]. Systematic studies of the existing data are revealing new reaction mechanisms, a rich diversity of nuclear structures involving weakly bound states coupled to an environment of scattering states and new competing decay modes that are challenging our understanding of the nucleus at its limits of stability. Such studies have provided not only the access to the basic properties of SHN: masses, lifetimes, reaction energies, emission rates, but also the possibility to predict decay properties and nuclear structure of not yet produced SHN.

Up to now, new significant results are obtained: new magic numbers associated with shell gaps at nonzero deformations, the coexistence of structures and shapes at different deformations at low excitations, the existence of isomeric states, and the possible pairing states at near the ground state.

In the present work, our main aim is to correlate on solid theoretical basis the decay data (energy levels, reactions energies, and half-lives) with the current information on the microscopic nuclear structure and the reaction decay mechanism.

\section{Formalism}

The approach we used here for studying the essential features of $\alpha$-decay of SHN is presented in Refs. [14, 15]. The first step is to match smoothly the four shell model wave functions of individual nucleons $\left(I_{n}^{k}(r)[S M]\right)$, which describe the shell model formation amplitude (SMFA) of the outgoing $\alpha$-particle (in channel $\mathrm{n}$ from the resonance

\footnotetext{
ae-mail: silist@theory.nipne.ro

b e-mail: claudia.anghel@ theory.nipne.ro
}

state k) with a general solution of the system of differential equations

$$
\begin{array}{r}
{\left[\frac{\hbar^{2}}{2 M}\left(\frac{d^{2}}{d r^{2}}-\frac{l(l+1)}{r^{2}}\right)-V_{n n}(r)+Q_{n}\right] u_{n}^{0(k)}(r)+} \\
+\sum_{m \neq n} V_{n m}(r) u_{m}^{0(k)}(r)=\left\{\begin{array}{c}
0 \\
I_{n}^{k[S M]}
\end{array}\right\} .
\end{array}
$$

The solutions $u_{n}^{0(k)}(r)$ describe the radial motion of the fragments at large and small separations, respectively, in terms of the reduced mass of the system $M$, the reaction energy $Q_{n}$, which includes screening energy, the angular momentum $l$, the formation amplitude (FA) $I_{n}^{k}(r)[S M]$, and the matrix elements of interaction potential $V_{n m}(r)$.

The shell model (SM) $\alpha$-particle FA is defined as the antisymmetrized projection of the parent wave function on the channel wave function

$$
I_{n}^{k}(r)[S M]=r\left\langle\Psi_{k}^{S M}\left(r_{i}\right) \mid \mathcal{A}\left[\Phi_{D}^{S M}\left(\eta_{1}\right) \Phi_{p}\left(\eta_{2}\right) Y_{l m}(\hat{r})\right]_{n}\right\rangle,
$$

where $\Phi_{D}^{S M}\left(\eta_{1}\right)$ and $\Phi_{p}\left(\eta_{2}\right)$ are the internal (space-spin) wave functions of the daughter nucleus and of the particle, $Y_{l m}(\hat{r})$ are the wave functions of the angular motion, $r$ connects the centers of mass of the fragments, and the symbol $<\mid>$ means integration over the internal coordinates and angular coordinates of relative motion. It should be pointed out that the spatial correlations imposed by Pauli principle on the nucleons in a simple shell model configuration are sufficient to determine the essential features of nuclear motion in the preformation stage. From the spectrum of single particle energies and wave functions we obtain the FA from active valence nucleons. The shell model overlap integral is estimated for the harmonic oscillator single particle wave functions of the parent and daughter nuclei. For SHN we use single particle proton states $1 i_{13 / 2}$, $2 f_{5 / 2}, 3 p_{3 / 2}$, and $3 p_{1 / 2}$ for $Z=102-120$ and single particle 
neutron states $2 g_{7 / 2}, 3 d_{5 / 2}, 3 d_{3 / 2}$ and $4 s_{1 / 2}$, for $N=150$ 178.

The matrix elements $V_{n m}(r)$ include nuclear and Coulomb components defined with the quadrupole $\left(\beta_{2}\right)$ and hexadecapole $\left(\beta_{4}\right)$ deformation parameters of the daughter nucleus. To avoid the usual ambiguities encountered in formulating the potential for the resonance tunneling of the barrier we iterate directly the nuclear potential in the equations of motion.

Solving Eq. (1) and Eq. (2) with minimal computational effort we get the reaction amplitude and the $\alpha$-decay width

$$
\Gamma_{n}^{k[S M]}=2 \pi\left|\frac{\int_{r_{\min }}^{r_{\max }} I_{n}^{k[S M]}(r) u_{n}^{0}(r) d r}{\int_{r_{\min }}^{r_{\max }} I_{n}^{k[S M]}(r) u_{n}^{k}(r) d r}\right|^{2}
$$

where the lower limit in the integrals is an arbitrary small radius $r_{\text {min }}>0$ while the upper limit $r_{\text {max }}$ is close to the first exterior node of $u_{n}^{0}(r)$.

Finally, from the systematics of calculated shell-model half-lives corrected by even-odd terms $h_{e-o}$ (even- $e$, odd$o$ ), a practical formula for the $\alpha$-decay half-lives has resulted [15]

$$
\begin{aligned}
& \log T_{\alpha}(\mathrm{s})=10.591\left(Z_{d}^{0.6} Q_{\alpha}^{-1 / 2}\right)-56.618, \quad r m s=0.078 \\
& (\mathrm{Z}=\mathrm{e}, \mathrm{N}=\mathrm{e})
\end{aligned}
$$

$\log T_{\alpha}(\mathrm{s})=10.148\left(Z_{d}^{0.6} Q_{\alpha}^{-1 / 2}\right)-53.386, \quad r m s=0.161$ $(\mathrm{Z}=\mathrm{e}, \mathrm{N}=\mathrm{o})$,

$$
\begin{aligned}
& \log T_{\alpha}(\mathrm{s})=10.148\left(Z_{d}^{0.6} Q_{\alpha}^{-1 / 2}\right)-53.386, \quad r m s=0.161 \\
& (\mathrm{Z}=\mathrm{o}, \mathrm{N}=\mathrm{e}),
\end{aligned}
$$

$$
\log T_{\alpha}(\mathrm{s})=10.225\left(Z_{d}^{0.6} Q_{\alpha}^{-1 / 2}\right)-53.797, \quad r m s=0.047
$$
$(\mathrm{Z}=\mathrm{o}, \mathrm{N}=\mathrm{o})$,

where $Q_{\alpha}$ is the reaction energy in $\mathrm{MeV}$ units and $Z_{d}$ is the atomic number of daughter nucleus. It was proven that the systematics of calculated SM half-lives well reproduces the systematics of available experimental data.

The SF-decay width is given by [16]

$$
\begin{aligned}
& \log T_{S F}(s)=1146.44-75.3153 Z^{2} / A+ \\
+ & 1.63792\left(Z^{2} / A\right)^{2}-0.0119827\left(Z^{2} / A\right)^{3}+ \\
+ & B_{f}\left(7.23613-0.0947022 Z^{2} / A\right)+H_{e-o},
\end{aligned}
$$

where $B_{f}$ is the SF barrier height (in $\mathrm{MeV}$ ) [17] and $H_{e-o}$ are new even-odd corrections [18]: $H_{e-o}=0$ for $(Z=e$, $N=e) ; 2.007$ for $(Z=e, N=o) ; 2.822$ for $(Z=o$, $N=e)$; and 3.357 for $(Z=o, N=o)$.

\section{Results}

The crucial quantity for a qualitative prediction of $\alpha$-decay half-life is the $Q_{\alpha}$-value that strongly affects the half-life

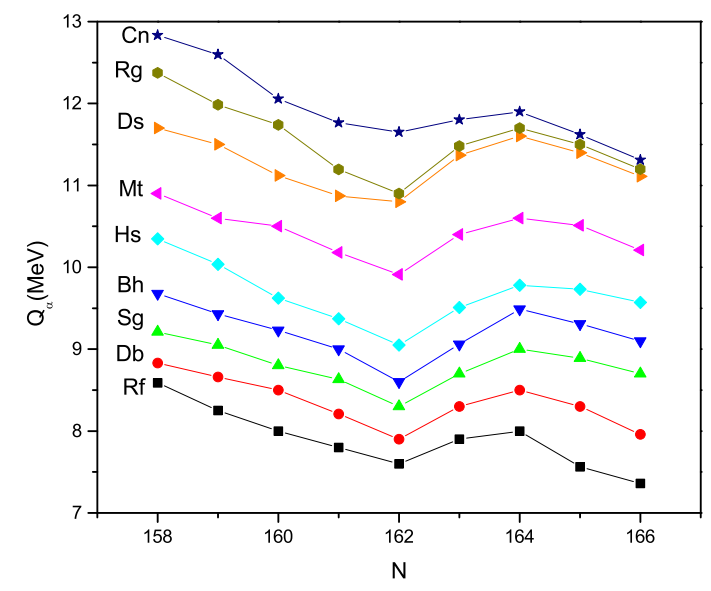

Figure 1. $Q_{\alpha}$ energy as a function of $N$ for the isotopic series of Ra-Cn, $Z=104-112$ and $N=158-166$.

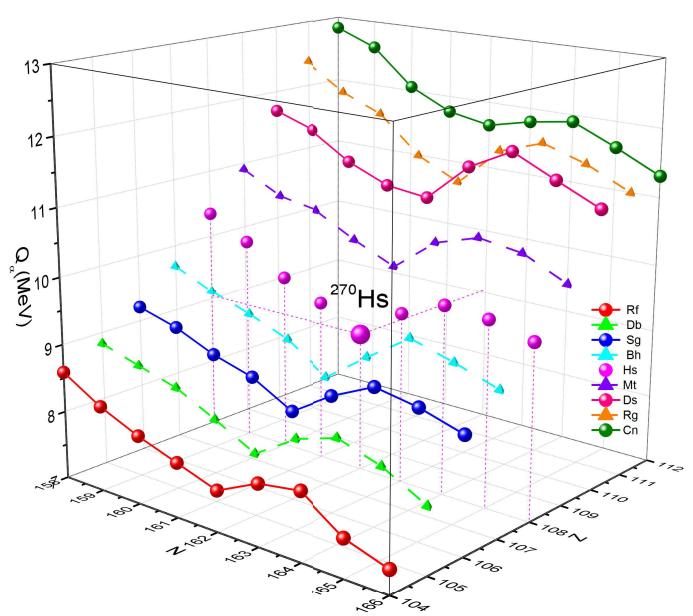

Figure 2. $Q_{\alpha}$ energy as a function of $Z$ and $N$ for the isotopic and isotonic series with $Z=104-112$ and $N=158-166$.

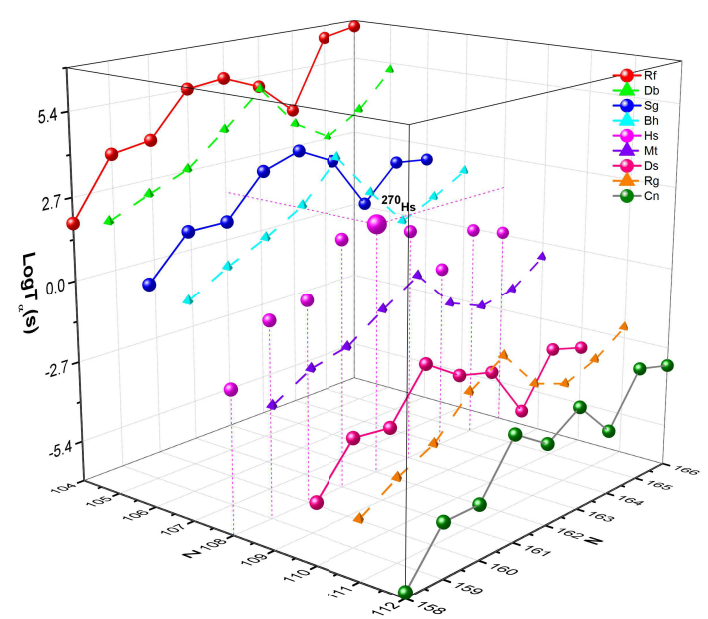

Figure 3. Calculated $\alpha$-half-lives as a function of $Z$ and $N$. Minimal $\alpha$-half-lives correspond to isotones $N=164$ and $N=160$ (only $Z=$ even). Maximal $\alpha$-half-lives correspond to isotones $N=161$, 163,165 . 


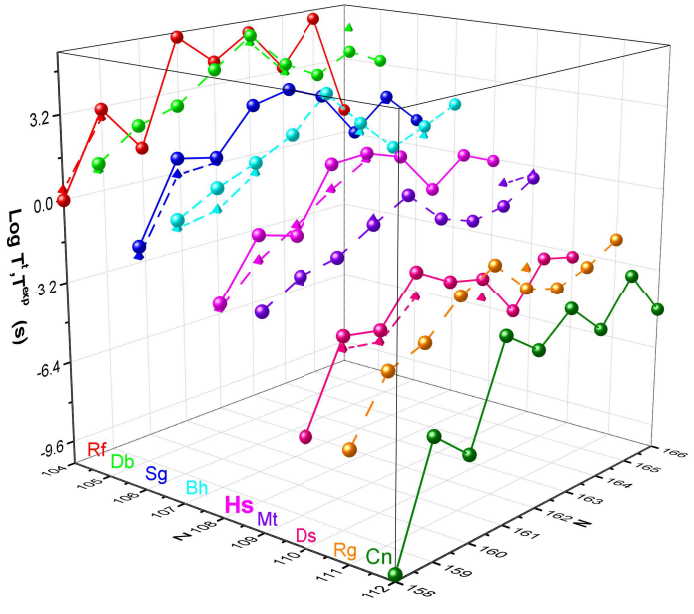

Figure 4. Experimental (triangles) and calculated (spheres) total half-lives of nuclei with $Z=104-112$ and $N=158-166$. Minimal values of calculated total half-lives are observed at $N=160$ isotones with $Z=$ even, where SF is the main decay channel, and at $N=164$ isotones, where the dominant process is $\alpha$-decay.

calculation due to the exponential decay law. To estimate the unknown $Q_{\alpha}$-values from the measured ones we employ the method [19], which takes into the account the effects of Coulomb energy and symmetry energy and validates not only the experimental measurements, but also predicts the $Q_{\alpha}$-values with a high accuracy. Once the parent or daughter nuclei have neutron or proton magic numbers (Fig. 1 and Fig. 2) the behavior of $Q_{\alpha}$-value is dramatically changed. Thus, the irregular behavior of $Q_{\alpha}$-value confirms the existence of the magic shells. The $Q_{\alpha}$-values in Fig. 1 and Fig. 2 present a linear decreasing trend for $N=158-162$ and $N=164-166$ and an increasing trend for $N=162-164$. Such a complicated trend was attributed [19] to the large symmetry energy that lowers the $Q_{\alpha}$-value at the magic shells (Fig. 1 and Fig. 2). In Fig. 2, we can see an increasing trend of $Q_{\alpha}$-value with increasing $Z$. Precise measurements and predictions of reaction energies are of importance for understanding of fine structure and transitions involving excited and isomeric states.

The $\alpha$-decay half-life (Fig. 3) varies approximately with $Q^{-1 / 2}$ and shows an irregular trend at the magic shells $Z=108$ and $N=162$. In Fig. 3, Fig. 5, and Fig. 6, one can see that the values $T_{\alpha}$ or the even-even nuclei are always shorter than those corresponding to the even-odd, oddeven, and odd-odd neighbors. Notice that by successive $\alpha$ decays the nuclear system becomes more and more stable. An example is the $\alpha$-decay chain ${ }^{274} \mathrm{Ds} \rightarrow{ }^{270} \mathrm{Hs} \rightarrow{ }^{266} \mathrm{Sg}$ (see Fig. 3), where the corresponding $T_{\alpha}$ values increase: $T_{\alpha}\left({ }^{274} \mathrm{Ds}\right)<T_{\alpha}\left({ }^{270} \mathrm{Hs}\right)<T_{\alpha}\left({ }^{266} \mathrm{Sg}\right)$. In Fig. 3, we can observe a weak $\alpha$-channel in isotonic sequence $N=160$ ( $Z=$ even), and a well defined $\alpha$-chain in $N=164$ sequence. Figure 4 shows our calculated total half-lives and some experimental results known up to date. Figure 4 reveals a main SF channel in $N=160$ ( $Z=$ even) sequence, and a main $\alpha$-decay channel in $N=164$ sequence. Comparing the results from Fig. 3 and Fig. 4 we may conclude that the SHN from unstable configurations undergo spontaneous $\alpha$-decay or SF until the nuclear stability is reached. Our results for the decay properties of SHN around ${ }^{270} \mathrm{Ds}$ are in good agreement with existing data [1-13]. In general, the calculated $\alpha$-decay half-lives are greater than the estimates based on previous predicted $Q_{\alpha}$-values of models developed in Refs. [14, 20-22]. Now, there are new improved parametrization of the unified model for $\alpha$-decay and $\alpha$ capture $[23,24]$ that may describe very well the available data. The agreement to the results of Refs. [25-30] for partial and total half-lives seems to be also good.

\section{Conclusions}

In this work we give a review of the experimental and calculated nuclear decay properties of nuclei around ${ }^{270} \mathrm{Hs}$ with $Z=104-112$ and $N=158-166$. New half-life predictions are made for many unknown nuclei from this region of nuclei. The main conclusions of the present study are summarized as follows:

- Nuclei from this region are typically unstable with respect to both $\alpha$-decay and SF.

- At ${ }^{270} \mathrm{Hs}$ the nuclear binding energy and the halflife increase strongly, while various reaction energies markedly decrease, these give rise to an increased stability.

- $\alpha$-decay dominates in nuclei over closed shells $Z>108$, $N>162\left({ }^{274} \mathrm{Ds}={ }^{270} \mathrm{Hs}+\alpha\right)$, while SF dominates in nuclei below the closed shells $\left({ }^{266} \mathrm{Sg}={ }^{270} \mathrm{Hs}-\alpha\right)$.

- Strong competition $\alpha$-SF is observed in $Z, N=$ even nuclei.

- $T_{S F}$ increases considerably due to the effect of unpaired nucleons and $T_{\alpha}$ is much less sensitive to the unpaired nucleons.

- The calculated half-lives are in good agreement with existing data.

We proved that the main nuclear decay properties can be well described with simple models based on the doubly magic core and the valence nucleons.

The experimental and theoretical studies of nuclear decay properties are providing a deep insight into the structure of SHN and stringent new tests of our theoretical understanding of decay processes at the limits of nuclear stability.

In brief, by studying the decay channels it is possible to inter-relate most nuclear species on the energy-time content basis and to determine the regions of greater stability. We showed that the decay rates can be predicted with a fair degree of confidence and this may help in synthesis and identification of new heaviest nuclei.

\section{References}

[1] S. Hofmann and G. Münzenberg, Rev. Mod. Phys. 72, 733 (2000)

[2] K. Morita et al., J. Phys. Soc. Jap. 73, 2593 (2004) 

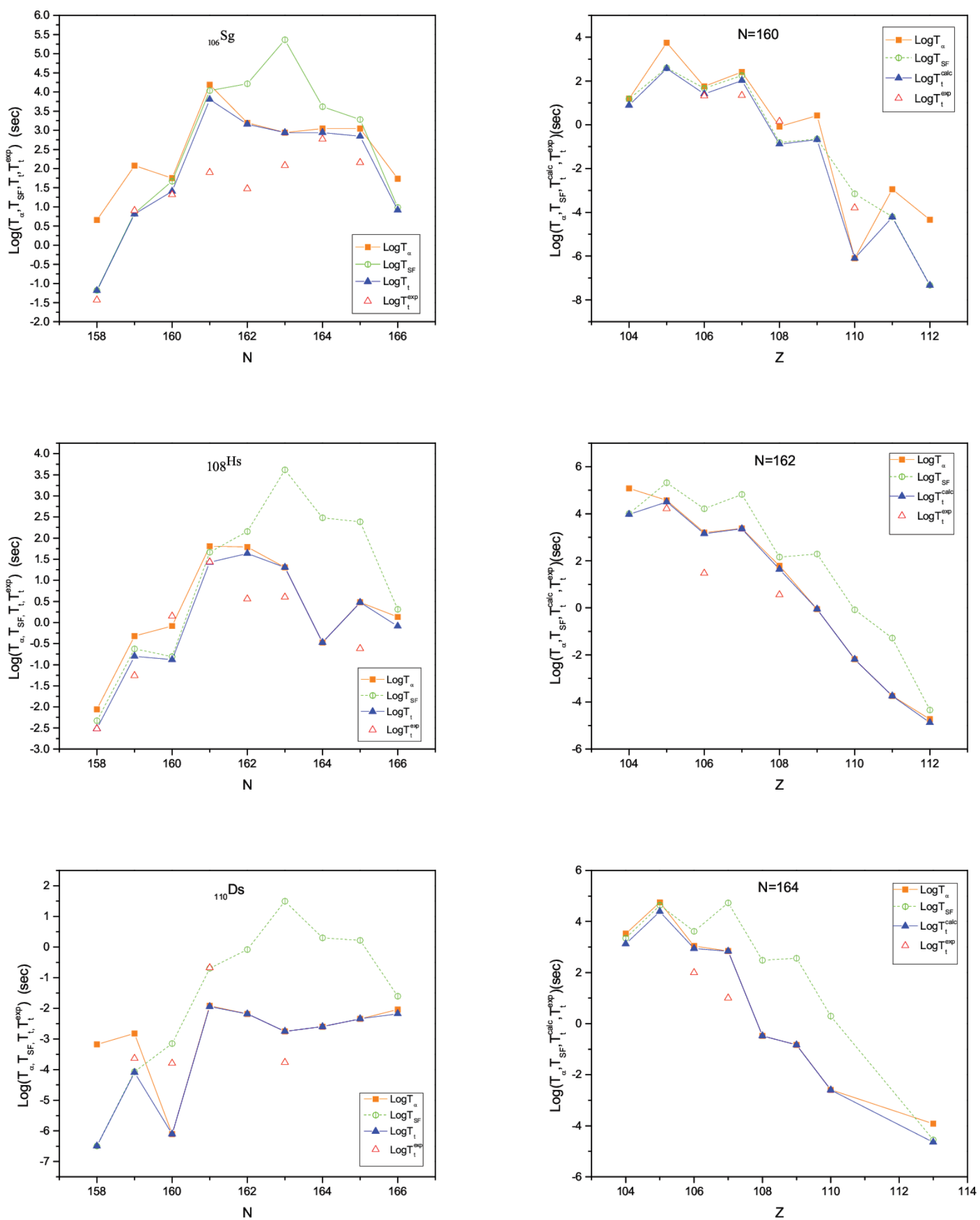

Figure 5. Experimental and calculated $\alpha$-decay, spontaneous fission, and total half-lives for isotopes of the elements $\mathrm{Sg}$, Hs and

Figure 6. Experimental and calculated $\alpha$-decay, spontaneous fission, and total half-lives for isotones with $\mathrm{N}=160,162,164$. 
[3] Yu.Ts. Oganessian et al., Phys. Rev. C 74, 044602 (2006)

[4] Yu.Ts. Oganessian et al., Phys. Rev. C 87, 054621 (2013)

[5] S. Hofmann et al., Eur. Phys. J. A 10, 5 (2001)

[6] I. Muntian, Z. Patyk, and A. Sobieczewski, Phys. Lett. B 500, 241 (2001)

[7] J. Dvorak et al., Phys. Rev. Lett. 97, 242501 (2006)

[8] J. Dvorak et al., Phys. Rev. Lett. 100, 132503 (2008)

[9] P.A. Ellison et al., Phys. Rev. Lett. 105, 182701 (2010)

[10] R. Graeger et al. Phys. Rev. C 81, 061601(R) (2010)

[11] A. Tuerler, Radiochimica Acta 100, 75 (2012)

[12] Yu.Ts. Oganessian et al., Phys. Rev. C 87, 034605 (2013)

[13] Chart of Nuclides, NNDC Brookhaven National Laboratory, http://www.nndc.bnl.gov/chart/

[14] A.I. Budaca and I. Silisteanu, Phys. Rev. C 88, 044618 (2013)

[15] I. Silisteanu and A.I. Budaca, At. Data Nucl. Data Tables 98, 1096 (2012)

[16] I.V. Karpov et al., Int. J. Mod. Phys. E 21, 1250013 (2012)

[17] P. Möller et al., Phys. Rev. C 79, 064304 (2009)
[18] I. Silisteanu and C.I. Anghel, Rom. J. Phys. 60, 444 (2015)

[19] J. Dong, W. Zuo, and W. Scheid, Phys. Rev. Lett. 107, 012501 (2011)

[20] A.I. Budaca and I. Silisteanu, J. Phys.: Conf. Series 413, 012027 (2013)

[21] A.I. Budaca and I. Silisteanu, J. Phys.: Conf. Series 337, 012022 (2012)

[22] I. Silisteanu and C.I. Anghel, Rom. J. Phys. 59, 724 (2014)

[23] S.P. Maydanyuk, P.-M. Zang, and S.V. Belchikov, Nucl. Phys. A 940, 89 (2015)

[24] V.Yu. Denisov, O.I. Davidovskaya, and I.Yu. Sedykh, Phys. Rev. C 92, 014602 (2015)

[25] O.V. Kiren, S.B. Gudennavar, and S.G. Bubbly, Rom. J. Phys. 57, 1335 (2012)

[26] K.P. Santhosh et al., J. Phys. G: Nucl. Part. Phys. 36, 115101 (2009)

[27] K.P. Santhosh and B. Priyanka, Phys. Rev. C 90, 054614 (2014)

[28] Yibin Qian and Zhongzhou Ren, Phys. Rev. C 90, 064308 (2014)

[29] Yibin Qian and Zhongzhou Ren, Phys. Lett. B 738, 87 (2014)

[30] R. Budaca, A. Sandulescu, and M. Mirea, Mod. Phys. Lett. A 30, 1550129 (2015) 